\title{
Disproportionate deficit in associative recognition relative to item recognition in global amnesia
}

\author{
KELLY SULLIVAN GIOVANELLO \\ Boston University School of Medicine, Boston, Massachusetts \\ MIEKE VERFAELLIE \\ Boston University School of Medicine, Boston, Massachusetts \\ and Boston VA Healthcare System, Boston, Massachusetts \\ and \\ MARGARET M. KEANE \\ Boston University School of Medicine, Boston, Massachusetts \\ and Wellesley College, Wellesley, Massachusetts
}

\begin{abstract}
In two experiments, we tested the hypothesis that medial temporal lobe (MTL) amnesic patients and, likewise, diencephalic (DNC) amnesic patients evidence a disproportionate deficit in memory for associations in comparison with memory for single items. In Experiment 1, we equated item recognition in amnesic and control participants and found that, under these conditions, associative recognition remained impaired both for MTL patients and for DNC patients. To rule out an alternative interpretation of the results of Experiment 1, in Experiment 2 we compared the performance of amnesic and control participants on a one-item recognition task and a two-item recognition task that required no memory for the association between members of word pairs. In the MTL group, when single-item recognition was equated to that of the controls, two-item nonassociative pair memory was equivalent as well. In the DNC group, nonassociative pair memory was impaired, but this impairment did not fully account for the impairment in associative memory. These findings indicate that memory for novel associations between items is disproportionately impaired in comparison with memory for single items in amnesia.
\end{abstract}

Global amnesia is a neurological syndrome characterized by a profound deficit in episodic memory as assessed by tasks of recall and recognition. This syndrome results from bilateral lesions centered in limbic and diencephalic brain structures. Several lines of research suggest that structures within the medial temporal lobe, particularly the hippocampus, play a critical role in binding or linking together unrelated pieces of information (Eichenbaum, Alvarez, \& Ramus, 2000; Fried, MacDonald, \& Wilson, 1997; Henke, Buck, Weber, \& Wieser, 1997; Mitchell, Johnson, Raye, \& D’Esposito, 2000; Yonelinas, Hopfinger, Buonocore, Kroll, \& Baynes, 2001). Thus, it has been proposed that the striking memory impairment observed in amnesic patients reflects a fundamental deficit in relational memory processing: the encoding and retrieval of relations or associations among informational elements (Eichenbaum \& Cohen, 2001). In accordance with this no-

This research was supported by National Institute of Mental Health Grants MH57681 and MH65113, National Institute of Neurological Disorders and Stroke Grant NS26985, and the Medical Research Service of the United States Department of Veterans Affairs. Correspondence concerning this article should be addressed to K. Sullivan Giovanello, Memory Disorders Research Center, Boston VA Healthcare System (151-A), 150 South Huntington Ave., Boston, MA 02130 (e-mail: ksull@bu.edu). tion, early studies of paired-associate learning in amnesia revealed that amnesic patients have profound difficulty in learning and remembering pairs of unrelated words (Cermak, 1976; Winocur \& Weiskrantz, 1976). To put it more broadly, episodic memories are formed by linking together the various cognitive, affective, and contextual components of an experience and, hence, make strong demands on the relational processing thought to be impaired in amnesia.

Episodic memory tasks vary in the extent to which they rely on relational processing. Isaac and Mayes (1999a, 1999b) have made a useful distinction between tasks that require the establishment of simple associations (i.e., associations between an item and a context that is kept constant across trials) and tasks that require the formation of more complex associations (i.e., associations that link together the informational elements that vary from trial to trial). This distinction is based on data from two sets of experiments in which rate of forgetting in normal and amnesic participants was examined. Whereas amnesic patients' free recall of prose and semantically related words declined at an accelerated rate, their recognition of the same material declined at a normal rate. Isaac and Mayes (1999a, 1999b) suggested that the differential forgetting rate observed between free recall and recognition reflected a difference in the extent to which each task depends on the retrieval of complex associative information. These re- 
sults remain open to alternative interpretations, however, since differences between recall and recognition can be framed in a number of different ways.

Memory for complex associations in amnesia has also been examined in the context of a recognition task in which participants study stimuli consisting of two elements (e.g., the words signal, barter, valley) and are then tested on old stimuli (e.g., signal) and conjunctions whose components are contained across several previously presented stimuli (e.g., barley). Importantly, both old and conjunction stimuli consist of previously presented stimulus elements. Therefore, successful discrimination between old and conjunction stimuli cannot be based on memory for individual stimulus elements, but must be based on memory for the associations formed between individual elements at study. The results from these studies demonstrate that amnesic patients are disproportionately impaired at rejecting conjunction stimuli (Kroll, Knight, Metcalfe, Wolf, \& Tulving, 1996; Reinitz, Verfaellie, \& Milberg, 1996; but see Stark \& Squire, 2003). Although these findings provide support for the notion that amnesia reflects an impairment of memory for associative information, the results do not provide direct evidence of a differential deficit in memory for complex associations (as opposed to simple associations), because no measure of memory for simple associations (i.e., the individual elements of which the stimuli were composed) was obtained.

The present study had two aims. The motivation for the first aim was the fact that recognition memory for simple associations and that for complex associations in amnesia have never been directly compared using identical encoding conditions. Here, we compared recognition memory for single items and for the association between items within the same task in patients with medial temporal lobe (MTL) damage. Second, we aimed to examine the performance of amnesic patients with diencephalic (DNC) damage. These patients do not have direct structural damage to the hippocampus, which is thought to be the critical substrate for binding or linking together previously unrelated pieces of information. However, it has been demonstrated that DNC patients show significant hypometabolism in the hippocampal formation (Fazio et al., 1992; Heiss, Pawlik, Holthoff, Kessler, \& Szelies, 1992). Therefore, we hypothesized that these patients, like MTL patients, would show a disproportionate impairment in associative relative to item memory. Such a finding would be in keeping with the extant literature indicating largely similar effects of MTL and DNC damage on the pattern of observed memory deficits (O'Connor \& Verfaellie, 2002).

To accomplish these aims, we conducted two experiments. In Experiment 1, we tested the hypothesis that amnesic patients with MTL lesions and, likewise, amnesic patients with DNC lesions have a disproportionate deficit in associative recognition in comparison with item recognition. In an effort to rule out an alternative hypothesis, in Experiment 2 we compared the performance of amnesic and nonamnesic individuals on a one-item recognition task and a two-item recognition task that requires no memory for the association between members of word pairs.

\section{EXPERIMENT 1}

The aim of Experiment 1 was to determine whether associative recognition of unrelated word pairs is disproportionately impaired in amnesia in comparison with item recognition. The typical procedure for comparing item and associative memory is to present participants with a study list of random word pairs that can be represented as $\mathrm{AB}, \mathrm{CD}, \mathrm{EF}, \mathrm{GH}, \ldots, \mathrm{KL}$. Item recognition involves the discrimination between items presented at study (e.g., A) and items not presented at study (e.g., P). Associative recognition involves distinguishing between intact pairs (e.g., CD) and rearranged pairs (e.g., EH). Importantly, both intact and rearranged pairs consist of two studied items. Therefore, successful discrimination between intact and rearranged pairs cannot be based on memory for individual items, but must be based on memory for the associations formed between individual items at study.

To assess whether amnesic patients have a disproportionate deficit in associative recognition, it is important to evaluate their performance under conditions in which their item recognition is similar to that of control participants. If amnesic patients and control participants perform at markedly different levels of item recognition, numerically similar differences between item and associative memory may not be functionally equivalent. To avoid this difficulty, we equated item recognition between amnesic and control participants and then examined whether, under these conditions, associative recognition was matched as well. Recognition was equated between groups by providing amnesic patients with additional study exposures. This method has been used in previous studies in which differential impairment in various forms of memory in amnesia was assessed (Chalfonte, Verfaellie, Johnson, \& Reiss, 1996; Giovanello \& Verfaellie, 2001). We predicted that associative recognition would be impaired in amnesia even under conditions in which item recognition is matched between groups, because associative recognition places stronger demands than does item recognition on relational processing - that is, processing thought to depend on the integrity of the hippocampus.

\section{Method}

Participants. Nine amnesic patients (6 men and 3 women) with damage to the MTL secondary to anoxia $(n=8)$ or encephalitis $(n=2)$ and 7 male patients with damage to the DNC secondary to alcoholic Korsakoff 's syndrome $(n=6)$ or bithalamic stroke $(n=1)$ participated in the experiment (see Table 1). All the patients with damage to the MTL, as well as the patient with damage to the DNC secondary to bithalamic stroke, had radiologically verified lesions. DNC damage secondary to alcoholic Korsakoff 's syndrome was presumed on the basis of previous reports (Jernigan, Shafer, Butters, \& Cermak, 1991; Victor, Adams, \& Collins, 1989). Eleven individuals ( 3 men and 8 women) without a history of alcoholism served as control participants for the MTL patients. Nine individuals $(5$ men and 4 women) with $(n=8)$ and without $(n=1)$ a history of alcoholism served as control participants for the DNC patients. The participants with a history of alcoholism had abstained from alcohol for at least 1 month prior to participation in the experiment. There were no differences between the MTL and DNC amnesic groups and their respective control groups in terms of age $[t(35)<1]$, education 
Table 1

Summary of Neuropsychological Characteristics of Amnesic Patients and Control Participants in Experiments 1 and 2

\begin{tabular}{|c|c|c|c|c|c|c|c|c|c|}
\hline \multirow[b]{2}{*}{ Patient } & \multirow[b]{2}{*}{ Etiology } & \multirow[b]{2}{*}{ Experiment } & \multirow{2}{*}{$\begin{array}{c}\text { Age } \\
\text { (Years) }\end{array}$} & \multicolumn{2}{|c|}{ WAIS-III } & \multicolumn{4}{|c|}{ WMS-III } \\
\hline & & & & Ed. & VIQ & GM & $\mathrm{AD}$ & VD & WM \\
\hline MTL01 & Anoxia & 1,2 & 63 & 20 & 111 & 52 & 64 & 56 & 83 \\
\hline MTL02 & Anoxia & 1 & 50 & 16 & 106 & 47 & 55 & 52 & 99 \\
\hline MTL03 & Anoxia & 1,2 & 72 & 18 & 122 & 75 & 80 & 72 & 102 \\
\hline MTL04 & Anoxia & 1,2 & 51 & 12 & 82 & 52 & 55 & 56 & 91 \\
\hline MTL05 & Anoxia & 1 & 37 & 16 & 86 & 49 & 52 & 53 & 93 \\
\hline MTL06 & Anoxia & 1,2 & 42 & 14 & 90 & 45 & 52 & 53 & 93 \\
\hline MTL07 & Anoxia & 1,2 & 44 & 14 & 111 & 59 & 52 & 72 & 96 \\
\hline MTL08 & Anoxia & 2 & 23 & 10 & 92 & 45 & 58 & 50 & 81 \\
\hline MTL09 & Encephalitis & 1,2 & 46 & 14 & 93 & 45 & 55 & 56 & 85 \\
\hline MTL10 & Encephalitis & 1,2 & 73 & 18 & 135 & 45 & 58 & 53 & 141 \\
\hline MTL11 & Encephalitis & 2 & 58 & 12 & 106 & 69 & 77 & 68 & 111 \\
\hline $\mathrm{DNC} 01$ & Bithalamic Stroke & 1,2 & 59 & 12 & 84 & 73 & 67 & 84 & 99 \\
\hline $\mathrm{DNC} 02$ & Korsakoff & 1,2 & 74 & 14 & 99 & 59 & 58 & 65 & 115 \\
\hline $\mathrm{DNC03}$ & Korsakoff & 1 & 71 & 12 & 77 & 72 & 74 & 65 & 96 \\
\hline DNC04 & Korsakoff & 2 & 80 & 14 & 105 & 66 & 64 & 62 & 121 \\
\hline DNC05 & Korsakoff & 1,2 & 59 & 16 & 92 & 47 & 58 & 56 & 85 \\
\hline DNC06 & Korsakoff & 1 & 56 & 12 & 97 & 66 & 74 & 62 & 108 \\
\hline $\mathrm{DNC} 07$ & Korsakoff & 1,2 & 82 & 9 & 100 & 72 & 74 & 75 & 91 \\
\hline DNC08 & Korsakoff & 1,2 & 51 & 18 & 111 & 69 & 64 & 72 & 81 \\
\hline DNC09 & Korsakoff & 2 & 77 & 14 & 103 & 72 & 71 & 68 & 115 \\
\hline MTL Controls & $(n=11)$ & 1 & 57.4 & 14.1 & 108.9 & & & & \\
\hline DNC Controls & $(n=9)$ & 1 & 64.8 & 13.6 & 104.2 & & & & \\
\hline MTL Controls & $(n=11)$ & 2 & 55.5 & 14.7 & 104.9 & & & & \\
\hline DNC Controls & $(n=9)$ & 2 & 69.1 & 13.8 & 104.7 & & & & \\
\hline
\end{tabular}

Note-WAIS-III, Wechsler Adult Intelligence Scale-third edition (Wechsler, 1997a); WMS-III, Wechsler Memory Scale-third edition (Wechsler, 1997b); Ed., education, in years; VIQ, verbal IQ; GM, general memory index; AD, auditory delay index; VD, verbal delay index; WM, working memory index; MTL, damage to the medial temporal lobe; DNC, damage to the diencephalic regions.

$[t(35)<1]$, or verbal IQ as measured by the Wechsler Adult Intelligence Scale-third edition [WAIS-III-Wechsler, 1997a; $t(35)=$ $1.5, p>.05]$.

Materials. Ninety-six low-frequency nouns $(M=20.3)$ consisting of one, two, or three syllables served as stimuli (Francis \& Kučera, 1982). Two word lists matched for frequency were constructed, each containing 48 words. Words from the first list were randomly paired with words from the second list to create 48 pairs, with the proviso that the resulting pairs be unrelated. Each of the 48 word pairs was assigned to one of four sets. Assignment of sets to the four test conditions (old item, new item, old pair, and recombined pair) was counterbalanced across participants. For old and new test items, one member of a word pair was chosen equally often from the right and from the left member of a pair. For recombined pairs, word pairs were recombined with other word pairs within the same set.

Procedure. During the study phase, the participants viewed 36 word pairs presented 1 pair at a time on a computer screen. As the pairs were presented, the experimenter read aloud a sentence that incorporated the two words in the pair. For each sentence, the participants were asked to rate on a scale of 1-5 the likelihood of occurrence of the information conveyed in the sentence. A card containing the likelihood values was kept in front of the participants throughout the study phase. Study word pairs remained on the computer screen until a likelihood judgment was given. The amnesic patients received 6 presentations of the study list, and the control participants received one presentation.

During the test phase, one or two words appeared on each of 48 trials. The 48 trials consisted of 12 old (studied) items, 12 new (unstudied) items, 12 old pairs, and 12 recombined pairs. In the event that one word appeared, the participants were instructed to decide whether or not that word had appeared on the study list. In the event that two words appeared, the participants were instructed to decide whether or not the two words constituted an old pair that had appeared previously during the study phase. The participants indicated their answers by pressing one of two buttons on a response box.

\section{Results and Discussion}

For each participant, the corrected measure of item recognition was the difference between the participants' "old" judgments to old items (i.e., hits) and his or her "old" judgments to new items (i.e., false alarms). The corrected measure of associative recognition was the difference between the participants' "old" judgments to old pairs (i.e., hits) and their "old" judgments to recombined pairs (i.e., false alarms). The amnesic and control participants' hit and false alarm rates for item and for associative recognition are shown in Table 2. Data are presented separately for MTL patients and for DNC patients. Mean corrected scores for item and for associative recognition are shown in Figure 1. A $2 \times 2 \times 2$ analysis of variance (ANOVA) with group (patient vs. control) and participant type (MTL vs. DNC) as between-participants variables and memory task (item recognition vs. associative recognition) as the within-participants variable was conducted on the corrected recognition scores. This analysis revealed a significant main effect of group $[F(1,32)=10.62, p<$ $.01]$ and a significant group $\times$ memory task interaction 
Table 2

Proportion of Studied and Unstudied Stimuli Endorsed as Old and Discriminability Scores $\left(d^{\prime}\right)$ for Item and Associative Recognition Tasks in Experiment 1 for Patients With

Structural Damage to the Medial Temporal Lobe (MTL) or Diencephalic Regions (DNC) and Respective Control Participants

\begin{tabular}{lrrrrr}
\hline & \multicolumn{2}{c}{ Amnesic } & & \multicolumn{2}{c}{ Control } \\
\cline { 2 - 3 } \multicolumn{1}{c}{ Task } & MTL & DNC & & MTL & DNC \\
\hline Item recognition & & & & \\
$\quad$ Studied & .77 & .77 & & .76 & .66 \\
$\quad$ Unstudied & .15 & .15 & & .06 & .16 \\
$\quad d$ ( & 1.85 & 1.94 & & 2.23 & 1.54 \\
Associative recognition & & & & \\
$\quad$ Studied & .73 & .78 & & .97 & .84 \\
$\quad$ Unstudied & .36 & .41 & & .08 & .11 \\
$d$ & 1.10 & 1.03 & & 2.91 & 2.29 \\
\hline
\end{tabular}

$[F(1,32)=25.31, p<.001]$, indicating that whereas item memory was matched, associative memory was lower in the amnesic patients. Also, whereas the control participants' associative memory was higher than their item memory, the reverse was true for the amnesic patients. No other main effects or interactions were significant (all $\left.F_{\mathrm{S}}<2.1\right)$.

We also analyzed these data using discriminability scores, and the results were identical. A $2 \times 2 \times 2$ ANOVA on $d$ ' scores revealed a significant main effect of group $[F(1,32)=10.24, p<.01]$ and a significant group $\times$ memory task interaction $[F(1,32)=29.05, p<.001]$. No other main effects or interactions were significant (all $\left.F_{\mathrm{S}}<1.95\right)$.

These results demonstrate that when item recognition is equated between amnesic and control participants by the provision of additional study exposures for amnesic participants, associative recognition remains impaired in amnesia. The finding of a disproportionate deficit in asso- ciative recognition in comparison with item recognition was observed for both MTL patients and DNC patients. These results are consistent with the notions that amnesia reflects a fundamental deficit in relational processing and that this deficit is related to the amount of relational processing required by a given task.

Although we observed no significant difference between the performance of the MTL patients and that of the DNC patients, the question nonetheless arises as to whether a disproportionate deficit in relational memory is observable in all amnesic patients or is a feature only of those patients with concomitant frontal pathology. To address whether frontal dysfunction was associated with associative memory performance, we calculated for each patient the difference between his or her discriminability scores $\left(d^{\prime}\right)$ for associative recognition and for item recognition and used this measure as an index of relational memory impairment. We then correlated this score with a composite frontal score consisting of the patient's mean rank on four measures derived from the Wisconsin card sorting task (number of categories and percent perseverative errors; Grant \& Berg, 1948), the controlled oral word association test (total number of appropriate responses; Benton \& Hamsher, 1989), and Trails B (reaction time; Spreen \& Strauss, 1991). This correlation was not significant $(r=.12)$. Therefore, our data do not provide support for the idea that the disproportionate deficit in relational memory is linked to frontal impairments.

It should be noted that the item and associative recognition tasks used in the present experiment differed not only in terms of the nature of the to-be-remembered information (items vs. associations between items), but also in terms of task load, because the item recognition task required judgments about single items, whereas the associative task required judgments about pairs of items. Before the present findings can be taken as evidence for a

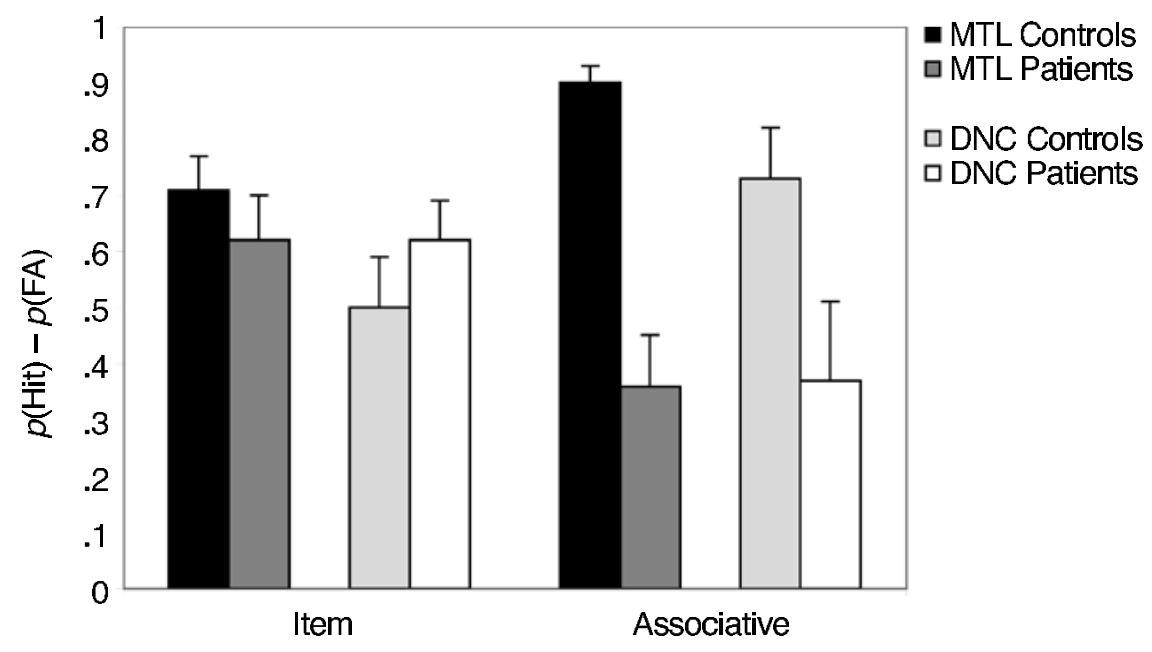

Figure 1. Mean corrected item and associative recognition scores for control participants and amnesic patients in Experiment 1. FA, false alarm. 
disproportionate impairment in associative memory, it is important to rule out the possibility that the impairment in the word pair condition was due to the high workload in that condition. In Experiment 2, we addressed this issue.

\section{EXPERIMENT 2}

In Experiment 2, we compared single-item recognition memory and two-item nonassociative pair memory in MTL patients and in DNC patients, as well as in their respective control participants. If the impairment in associative memory observed in Experiment 1 was due to the greater task load associated with making recognition judgments about two items, then when item memory in amnesic and control groups is equated, nonassociative memory for word pairs should still be impaired. Alternatively, if, as we hypothesized, the previously observed impairment in associative recognition reflects a true deficit in associative memory, then nonassociative memory for word pairs should be similar across groups under conditions in which item memory is matched.

\section{Method}

Participants. Nine amnesic patients (6 men and 3 women) with damage to the MTL secondary to anoxia $(n=6)$ or encephalitis $(n=3)$ and seven amnesic patients (6 men and 1 woman) with damage to the DNC secondary to alcoholic Korsakoff's syndrome $(n=6)$ or bithalamic stroke $(n=1)$ participated in the experiment (see Table 1). All the patients with damage to the MTL, as well as the patient with damage to the DNC secondary to bithalamic stroke, had radiologically verified lesions. DNC damage secondary to alcoholic Korsakoff's syndrome was presumed on the basis of previous reports (Jernigan et al., 1991; Victor et al., 1989). Eleven individuals ( 2 men and 9 women) without a history of alcoholism served as control participants for the MTL patients. Nine individuals ( 6 men and 3 women) with $(n=8)$ and without $(n=1)$ a history of alcoholism served as control participants for the DNC patients. The participants with a history of alcoholism had abstained from alcohol for at least 1 month prior to participation in the experiment. There were no differences between the MTL and DNC amnesic groups and their respective control groups in terms of age $[t(35)<1]$, education $[t(35)<1]$, or WAIS-III verbal IQ $[t(35)<1]$.

Materials. One hundred forty-four low-frequency nouns $(M=$ 21.2) consisting of one, two, or three syllables served as stimuli (Francis \& Kučera, 1982). Two word lists matched for frequency were constructed, each containing 72 words. Words from the first list were randomly paired with words from the second list to create 72 pairs, with the proviso that the resulting pairs be unrelated. Each of the 72 word pairs was assigned to one of six sets. Assignment of sets to the five test conditions (old item, new item, recombined pair, old/new pair, and new pair) was counterbalanced across participants. For old and new test items, one member of a word pair was chosen equally often from the right and from the left member of a pair. For recombined pairs, word pairs were recombined with other word pairs within the same set. For old/new pairs, one member of the pair was chosen from a studied set, and the other member was chosen from an unstudied set.

Procedure. The procedure during the study phase was identical to that in Experiment 1. During the test phase, one or two words appeared on each of 60 trials. The 60 trials were composed of 12 old items, 12 new items, 12 recombined pairs, 12 old/new pairs, and 12 new pairs. In the event that one word appeared, the participants were instructed to decide whether or not that word had appeared on the study list. In the event that two words appeared, the participants were instructed to decide whether or not both words had been seen on the study list. The participants were further instructed that it was insignificant whether or not the two words had appeared together previously. Old/new pairs were included in the experiment to ensure that the participants based their recognition judgments on both items of the pair, but these trials were not included in the data analysis. The participants indicated their answers by pressing one of two keys on a response box.

\section{Results and Discussion}

For each participant, the corrected measure of item recognition was the difference between the participant's "old" judgments to old items (i.e., hits) and his or her "old" judgments to new items (i.e., false alarms). The corrected measure of nonassociative pair recognition was the difference between the participants" "old" judgments to recombined pairs (i.e., hits) and their "old" judgments to new pairs (i.e., false alarms). The amnesic and control participants' hit and false alarm rates for item and for nonassociative pair recognition are shown in Table $3 .^{1}$ Data are presented separately for the MTL patients and for the DNC patients. Mean corrected scores for item and for nonassociative pair recognition are shown in Figure 2. A $2 \times 2 \times 2$ ANOVA with group (patient vs. control) and participant type (MTL vs. DNC) as between-participants variables and memory task (item recognition vs. nonassociative pair recognition) as a within-participants variable was conducted on the corrected recognition scores. This analysis revealed a significant main effect of group only $[F(1,32)=12.21, p<.01]$. No other main effects or interactions were significant (all $F_{\mathrm{s}}<1.5$ ). The absence of a significant main effect of participant type indicates that there was no difference between groups based on site of damage. Furthermore, the absence of a group $\times$ memory task interaction indicates that the amnesic patients were equally impaired in item and nonassociative pair memory.

The results of the signal detection analysis revealed a somewhat more complex pattern. A $2 \times 2 \times 2$ ANOVA on $d^{\prime}$ scores revealed a significant main effect of group $[F(1,32)=10.87, p<.01]$ as well as a marginally signif-

Table 3

Proportion of Studied and Unstudied Stimuli Endorsed as Old and Discriminability Scores $\left(d^{\prime}\right)$ for Item and Nonassociative Pair Recognition Tasks in Experiment 2 for Patients With Structural Damage to the Medial Temporal Lobe (MTL) or Diencephalic Regions (DNC) and Respective Control Participants

\begin{tabular}{|c|c|c|c|c|}
\hline \multirow[b]{2}{*}{ Task } & \multicolumn{2}{|c|}{ Amnesic } & \multicolumn{2}{|c|}{ Control } \\
\hline & MTL & DNC & MTL & $\mathrm{DNC}$ \\
\hline \multicolumn{5}{|l|}{ Item recognition } \\
\hline Studied & .82 & .93 & .89 & .84 \\
\hline Unstudied & .27 & .31 & .08 & .14 \\
\hline$d$ & 1.63 & 2.08 & 2.65 & 2.11 \\
\hline \multicolumn{5}{|c|}{ Nonassociative pair recognition } \\
\hline Studied & .79 & .83 & .85 & .88 \\
\hline Unstudied & .21 & .26 & .05 & .06 \\
\hline$d$ & 1.81 & 1.82 & 2.56 & 2.62 \\
\hline
\end{tabular}




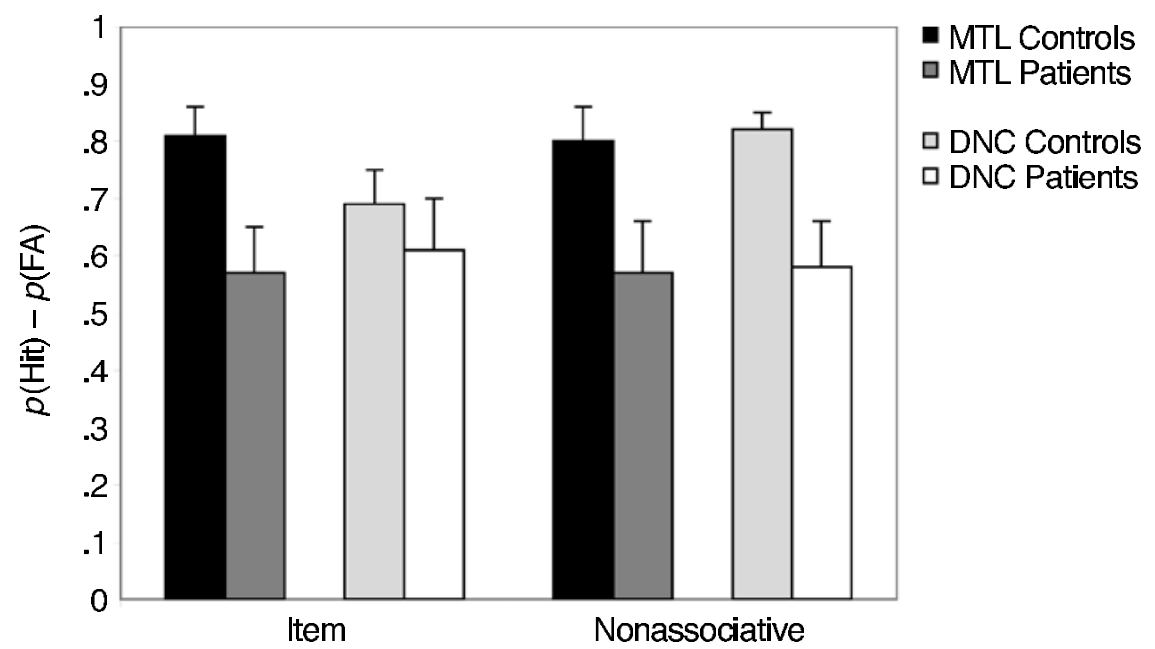

Figure 2. Mean corrected item and nonassociative pair recognition scores for control participants and amnesic patients in Experiment 2. FA, false alarm.

icant group $\times$ participant $\times$ memory task interaction $[F(1,32)=3.42, p=.07]$. No other main effects or interactions were significant (all $F_{\mathrm{s}}<1.38$ ). The marginally significant three-way interaction indicates that a different pattern of performance emerged for MTL patients and for DNC patients. For this reason, the two patient groups are discussed separately.

Analysis of discriminability scores $\left(d^{\prime}\right)$ for the MTL patients revealed a main effect of group $[F(1,18)=8.35$, $p<.01]$. Neither the main effect of memory task nor the group $\times$ memory task interaction was significant (both $F_{\mathrm{s}}<1$ ), indicating that there was no disproportionateimpairment in nonassociative pair memory for MTL patients. However, it is difficult to interpret these results, because item recognition was not equivalent between the MTL patients and the respective control participants. To create two subgroups for which item memory was equivalent $($ MTL patients $=2.0$, MTL controls $=2.1$ ), we selected the MTL patients with the highest item memory $(n=6)$ and the MTL control participants with the lowest item memory $(n=6)$. Nonassociative pair memory was 2.0 and 2.3 for the MTL patient and control subgroups, respectively. Again, the group $\times$ memory task failed to reach significance $(F<1)$, confirming that MTL patients were not disproportionately impaired in nonassociative pair memory.

Returning to the DNC patients, item recognition was equivalent between the patients and their controls, but their performance did suffer as task load increased, as is indicated by a marginally significant group $\times$ memory task interaction $[F(1,14)=3.08, p=.10]$. A critical question, however, is whether the associative deficit can be attributed entirely to a deficit in making judgments about two items. We addressed this question by performing a threeway ANOVA comparing the performance of DNC patients and that of the corresponding control participants on single-item and two-item tasks across the two experiments. This analysis, conducted with patients $(n=5)$ and controls $(n=7)$ who had participated in both experiments, revealed a marginally significant three-way interaction $[F(1,20)=2.83, p=.10]$. Follow-up analyses indicated that there was no difference in the DNC patients' item memory between the two experiments (group $\times$ memory task interaction, $F<1$ ), but that in the DNC patients the magnitude of impairment was greater for associative memory (Experiment 1 ) than for nonassociative pair memory [Experiment 2; group $\times$ memory task interaction, $F(1,10)=5.86, p<.05]$. The lack of significance in the three-way interaction likely reflects a lack of power due to the reduced number of participants, since combining the data from the two amnesic subgroups and the two control subgroups that had participated in both experiments yielded a significant three-way interaction $[F(1,42)=$ $4.43, p<.05]$. Therefore, the associative deficit in amnesia observed in Experiment 1 is not due solely to the higher task load associated with making judgments about two items.

\section{GENERAL DISCUSSION}

In the present study, we tested the hypothesis that MTL amnesic patients and, likewise, DNC amnesic patients have a disproportionate deficit in memory for associations between words (associative recognition) in comparison with memory for single words (item recognition). In Experiment 1 , we equated item recognition in amnesic and control groups and examined whether, under these conditions, associative recognition was equivalent. Because associative recognition depends on relational processing to a greater extent than does item recognition, we hypothesized that associative recognition would be disproportionately impaired in amnesia. As we predicted, when item 
recognition was equated between amnesic and control participants, the amnesic patients' associative recognition remained impoverished. The finding of a disproportionate deficit in associative recognition in comparison with item recognition was observed for both the MTL patients and the DNC patients, suggesting that even though the structural damage is distinct in these two patient groups, the functional impairment is equivalent.

In Experiment 2, we sought to rule out the possibility that the deficit in associative memory observed in Experiment 1 was due to the greater task load associated with making recognition judgments about two items. To this end, we compared single-item recognition memory and two-item nonassociative recognition memory in amnesic and control groups. Under conditions in which single-item recognition was equated between groups, two-item nonassociative memory was nonsignificantly lower in the MTL amnesic group. The DNC patients' performance did suffer as task load increased, possibly because damage to frontal regions disrupts the mechanisms responsible for the maintenance and coordination of multiple concurrent activities (Cowey \& Green, 1996). However, as can be seen in a comparison across experiments, the DNC patients performed equivalently on the item memory tasks and performed significantly worse on the associative recognition task than on the nonassociative pair task. These results demonstrate that the deficit in associative recognition observed in Experiment 1 was not solely an artifact of task load, but reflected a disproportionate impairment in associative memory.

Our findings differ from those of a recent report that showed similar impairments in recognition memory for single items and for associations in patients with damage to the hippocampal region (Stark, Bayley, \& Squire, 2002). However, several points deserve mention. First, as was noted by Stark et al., amnesic patients' associative recognition performance was not reliably above chance in their Experiment 1. Second, in their Experiment 2, in which amnesics' item recognition was equated to that of the control participants, the amnesics' item recognition appeared to be limited by ceiling effects. Such ceiling effects could mask an impairment in associative recognition memory in amnesia.

The results of the present study may seem inconsistent with the findings of a recent functional imaging study that demonstrated equivalent hippocampal activation during item recognition and associative recognition (Stark \& Squire, 2001). However, the nature of the associative recognition task used in the imaging study differed considerably from that in the present study. Specifically, participants in the imaging experiment were administered an associative recognition task in which pictures of nameable objects were presented at study and the names of these objects were presented during the subsequent recognition test. Thus, those participants had to retrieve a preexisting association between an object and its name. In contrast, the participants in our Experiment 1 studied previously unrelated word pairs that required the formation of a novel link and were subsequently tested on their ability to retrieve this newly formed association. It is likely that the difference in the nature of the associative recognition task led to the divergent results.

The deficit in associative memory reported in the present study was observed using associations that were established between items or components of a similar kind (i.e., within-domain associations). It should be noted, however, that there are two reports of patients with lesions restricted to the hippocampus who showed intact withindomain associations. Vargha-Khadem et al. (1997) described three children with selective hippocampal damage who demonstrated normal associative recognition for word pairs and pairs of faces. Likewise, Mayes et al. (2001) have reported intact associative recognition for word pairs and pairs of faces in a patient with adult-onset amnesia (Y.R.), whose damage was limited to the hippocampus. These findings suggest that damage restricted to the hippocampus spares within-domain associations and raise the possibility that a subset of our patients, in whom selective hippocampal damage has occurred, may show a proportionate deficit in associative memory relative to item memory.

To determine whether the extent of MTL damage influenced associative recognition performance in the present study, we performed an analysis on a subgroup of patients $(n=3)$ with lesions restricted to the hippocampus (i.e., CA fields, dentate gyrus, and subiculum) and a subgroup of patients $(n=6)$ in whom lesions extended beyond the hippocampus to include the surrounding cortices (i.e., entorhinal, perirhinal, and parahippocampal cortices). The extent of MTL damage was determined by a neurologist on the basis of visual inspection of the MRI data for each patient. Mean item recognition for the restricted hippocampal lesion group was .75 $(S D=.30)$, whereas that group's mean associative recognition was $.53(S D=.19)$. For the more extensive MTL lesion group, mean item recognition was $.55(S D=.21)$ and mean associative recognition was $.28(S D=.26)$. An ANOVA revealed a main effect of stimulus type $[F(1,6)=8.28, p<.03]$ but no main effect of group $[F(1,6)=1.67]$ and no interaction $[F(1,6)<1]$. These findings need to be interpreted with caution, since the patients with restricted hippocampal lesions performed numerically better than the patients with extensive MTL lesions. Nonetheless, the patterns of performance were similar in the two patient groups-better item recognition than associative recognition - and distinct from that in the control groups, in whom associative recognition was better than item recognition. These findings do not support the notion that damage restricted to the hippocampus spares memory for within-domain associations.

One possible explanation for the discrepancy between the present findings and those reported in patients with lesions restricted to the hippocampus (described above) concerns the effect of test format. Whereas in our study a yes/no associative recognition test was employed, in those studies in which intact within-domain associations were 
observed, a forced-choice associative recognition test was used. Norman and O'Reilly (in press) have recently developed a neural network model of recognition memory that predicts that patients with hippocampal damage should perform better on forced-choice recognition than on yes/no recognition due to the greater contribution of familiaritybased recognition to the former than to the latter test format. Thus, test format may be a potential determinant of the extent to which associative recognition is impaired relative to item recognition in patients with lesions restricted to the hippocampus.

Thus far, we have posited that the disproportionate deficit in associative recognition in amnesia reflects a deficit in relational memory processing. It is also possible, however, that it reflects a disproportionate deficit in recollection relative to familiarity, since associative recognition is based on conscious recollection to a greater extent than is item recognition, and, conversely, that item memory is based on familiarity to a greater extent than is associative recognition (Hockley \& Consoli, 1999). Other studies, however, have demonstrated deficits in relational memory processing that cannot be accounted for by the recollection/ familiarity distinction (Chun \& Phelps, 1999; Ryan, Althoff, Whitlow, \& Cohen, 2000). Therefore, although we cannot rule out the possibility that the demands on recollection were responsible for the amnesics' disproportionate impairment on the associative recognition task, a fundamental deficit in relational processing provides a fuller account of the performance in amnesia across paradigms.

\section{REFERENCES}

Benton, A. L., \& Hamsher, K. D. (1989). Multilingual aphasia examination. Iowa City, IA: AJA Associates.

Cermak, L. (1976). The encoding capacity of a patient with amnesia due to encephalitis. Neuropsychologia, 14, 311-326.

Chalfonte, B. L., Verfaellie, M., Johnson, M. K., \& Reiss, L. (1996). Spatial location memory in amnesia: Binding item and location information under incidental and intentional encoding conditions. Memory, 4, 591-614.

Chun, M. M., \& Phelps, E. A. (1999). Memory deficits for implicit contextual information in amnesic subjects with hippocampal damage. Nature Neuroscience, 2, 844-847.

Cowey, C. M., \& Green, S. (1996). The hippocampus: A "working memory" structure? The effect of hippocampal sclerosis on working memory. Memory, 4, 19-30.

Eichenbaum, H., Alvarez, P., \& Ramus, S. J. (2000). Animal models of amnesia. In L. S. Cermak (Ed.), Memory and its disorders (2nd ed., Vol. 2, pp. 1-24). Amsterdam: Elsevier.

Eichendaum, H., \& Cohen, N. J. (2001). From conditioning to conscious recollection: Memory systems of the brain. New York: Oxford University Press.

Fazio, F., Perani, D., Gilardi, M. C., Colombo, F., Cappa, S. F., Vallar, G., Bettinardi, V., Paulesu, E., Alberoni, M., Bressi, S., Franceschi, M., \& Lenzi, G. L. (1992). Metabolic impairment in human amnesia: A PET study of memory networks. Journal of Cerebral Blood Flow \& Metabolism, 12, 353-358.

Francis, W. N., \& KuČERA, H. (1982). Frequency analysis of English usage: Lexicon and grammar. Boston: Houghton Mifflin.

Fried, I., MacDonald, K. A., \& Wilson, C. L. (1997). Single neuron activity in human hippocampus and amygdala during recognition of faces and objects. Neuron, 18, 753-765.

Giovanello, K. S., \& Verfaellie, M. (2001). The relationship between recall and recognition in amnesia: Effects of matching recogni- tion between patients with amnesia and controls. Neuropsychology, 15, 444-451.

Grant, D. A., \& Berg, E. A. (1948). A behavioral analysis of the degree of reinforcement and ease of shifting to new responses in a Weigl-type card sorting problem. Journal of Experimental Psychology: General, 38, 404-411.

Heiss, W. D., Pawlik, G., Holthoff, V., Kessler, J., \& Szelies, B. (1992). PET correlates of normal and impaired memory function. Cerebrovascular Brain Metabolism Review, 4, 1-27.

Henke, K., Buck, A., Weber, B., \& Wieser, H. G. (1997). Human hippocampus establishes associations in memory. Hippocampus, 7, 249256.

Hockley, W. E., \& Consoli, A. (1999). Familiarity and recollection in item and associative recognition. Memory \& Cognition, 27, 657-664.

ISAAC, C. L., \& MAYES, A. R. (1999a). Rate of forgetting in amnesia: I. Recall and recognition of prose. Journal of Experimental Psychology: Learning, Memory, \& Cognition, 25, 942-962.

IsAAC, C. L., \& MAYES, A. R. (1999b). Rate of forgetting in amnesia: II. Recall and recognition of word lists at different levels of organization. Journal of Experimental Psychology: Learning, Memory, \& Cognition, 25, 963-977.

Jernigan, T. L., Shafer, K., Butters, N., \& Cermak, L. S. (1991). Magnetic resonance imaging of alcoholic Korsakoff patients. Neuropsychopharmacology, 4, 175-186.

Kroll, N. E. A., Knight, R. T., Metcalfe, J., Wolf, E. S., \& TulVING, E. (1996). Conjunction failure as a source of memory illusions. Journal of Memory \& Language, 35, 176-196.

Mayes, A. R, Isaac, C. L., Holdstock, J. S., Hunkin, N. M., Montaldi, D., Downes, J. J., MacDonald, C., Cezayirli, E., \& Roberts, J. N. (2001). Memory for single items, word pairs, and temporal order of different kinds in a patient with selective hippocampal lesions. Cognitive Neuropsychology, 18, 97-123.

Mitchell, K. J., Johnson, M. K., Raye, C. L., \& D’Esposito, M. (2000). fMRI evidence of age-related hippocampal dysfunction in feature binding working memory. Cognitive Brain Research, 10, $197-$ 206.

Norman, K. A., \& O'Reilly, R. C. (in press). Modeling hippocampal and neocortical contributions to recognition memory: A complementary learning systems approach. Psychological Review.

O'Connor, M., \& Verfaellie, M. (2002). The amnesic syndrome: Overview and subtypes. In A. Baddeley, M. D. Kopelman, \& B. A. Wilson (Eds.), The handbook of memory disorders (pp. 145-166). Chichester, U.K.: Wiley.

Reinitz, M. T., Verfaellie, M., \& Milberg, W. P. (1996). Memory conjunction errors in normal and amnesic subjects. Journal of Memory \& Language, 35, 286-299.

Ryan, J. D., Althoff, R. R., Whit low, S., \& Cohen, N. J. (2000). Amnesia is a deficit in relational memory. Psychological Science, 11, 454-461.

Spreen, O., \& Strauss, E. (1991). A compendium of neuropsychological tests. New York: Oxford University Press.

Stark, C. E., Bay ley, P. J., \& SQuire, L. R. (2002). Recognition memory for single items and for associations is similarly impaired following damage to the hippocampal region. Learning \& Memory, 9, 238242.

Stark, C. E., \& Squire, L. R. (2001). Simple and associative recognition memory in the hippocampal region. Learning \& Memory, 8, 190197.

Stark, C. E., \& Squire, L. R. (2003). Hippocampal damage equally impairs memory for single items and memory for conjuctions. Hippocampus, 13, 281-292.

Vargha-Khadem, F., Gadian, D. G., Watkins, K. E., Connelly, A., Van Paesschen, W., \& Mishrin, M. (1997). Differential effects of early hippocampal pathology on episodic and semantic memory. Science, 277, 376-380.

Victor, M., Adams, R. D., \& Collins, G. H. (1989). The WernickeKorsakoff syndrome and related neurologic disorders due to alcoholism and malnutrition (2nd ed.). Philadelphia: Davis.

WECHSLER, D. (1997a). Wechsler Adult Intelligence Scale-Third edition: Administration and scoring manual. San Antonio, TX: Psychological Corp. 
WeCHSLER, D. (1997b). Wechsler Memory Scale-Third edition: Administration and scoring manual. San Antonio, TX: Psychological Corp.

Winocur, G., \& WeISKRANTZ, L. (1976). An investigation of pairedassociate learning in amnesia. Neuropsychologia, 14, 97-110.

Yonelinas, A. P., Hopfinger, J. B., Buonocore, M. H., Kroll, N. E. A., \& BAYNES, K. (2001). Hippocampal, parahippocampal and occipital-temporal contributions to associative and item recognition memory: An f MRI study. NeuroReport, 12, 359-363.

\section{NOTE}

1. In Experiment 2, mean accuracy scores for old/new pairs were .67 (MTL patients), .55 (DNC patients), .88 (MTL controls), and .69 (DNC controls). To examine whether the participants had based their decisions on both words of a pair, we compared the observed proportion of "old" responses to old/new pairs with that which could be expected on the basis of the known probability of "old" responses to old item pairs and to new item pairs. Expected proportions were calculated as the product of the square root of the known probability of "old" responses to old item pairs and the square root of the known probability of "old" responses to new item pairs. The expected and observed probabilities did not significantly differ from each other in either the amnesic patients $\left(\chi^{2}=1.11\right.$, n.s.) or the control participants $\left(\chi^{2}<1\right.$, n.s.), suggesting that both the amnesic patients and the control participants had based their judgments on the evaluation of both words in the pair.

(Manuscript received December 10, 2002; revision accepted for publication August 22, 2003.) 